PROCEEDINGS OF THE

AMERICAN MATHEMATICAL SOCIETY

Volume 37, Number 1, January 1973

\title{
EMBEDDING OF CATEGORIES
}

\author{
MICHAEL BARR
}

Dedicated to the memory of George S. Rinehart

\begin{abstract}
In this paper we generalize the notion of exact functor to an arbitrary category and show that every small category has a full embedding into a category of all set-valued functors on some small category. The notion of exact is such that this result generalizes the author's exact embedding of regular categories and, indeed, Mitchell's embedding of abelian categories. An example is given of the type of diagram-chasing argument that can be given with this embedding.
\end{abstract}

Introduction. In [Barr] we proved that every small regular category has an exact embedding into a set-valued functor category $(C, S)$ where $C$ is some small category. In this paper we show that the same result can be proved for any small category when the definition of exact functor is slightly extended. This new definition will agree with the previous one when the category is regular.

The embedding which results can be used to chase diagrams in completely arbitrary categories, much as Mitchell's theorem does for abelian categories. As an illustration of this, we derive a theorem of Grothendieck on the descent of pullbacks.

1. Universal regular epimorphisms. In any category a map is called a regular epimorphism if it is the coequalizer of some parallel pair of maps. One usually defines $f: X \rightarrow Y$ to be a universal regular epimorphism if for every $Y^{\prime} \rightarrow Y$, the fibred product $Y^{\prime} \times_{Y} X$ exists and the projection $Y^{\prime} \times_{Y} X \rightarrow Y^{\prime}$ is always a regular epimorphism. It is gradually becoming clearer that the universal regular epimorphisms are the "good" epimorphisms. In fact a good case could be made that these should be termed the quotient mappings. In a regular category, every regular epimorphism is universally so.

In [Verdier] it is shown how every category $\boldsymbol{X}$ has a full embedding into a topos $E$ where $E$ is the category of sheaves for the so-called canonical topology on $\boldsymbol{X}$. A brief description of $\boldsymbol{E}$ and the embedding $R$ follows.

Received by the editors May 12, 1972.

AMS (MOS) subject classifications (1970). Primary 18A25, 18B15.

Key words and phrases. Exact embedding, universal regular epimorphism, sheaves.

(c) American Mathematical Society 1973 
Let $R: X \rightarrow\left(X^{\mathrm{op}}, S\right)$ be the Yoneda embedding into the indicated functor category. A subfunctor $F \mapsto R X$ is called a cover of $X$ if for all $f: X_{1} \rightarrow X$ and all $Y \in X$, the map induced by the projection

$$
\left(R X_{1}, R Y\right) \rightarrow\left(F \times_{R X} R X_{1}, R Y\right)
$$

is an isomorphism. In particular, by taking $f=X$ (=identity map of $X$ ), we see that $(R X, R Y) \rightarrow(F, R Y)$ is required to be an isomorphism. A sheaf is defined to be a functor $E$ such that the natural map

$$
E X \cong(R X, E) \rightarrow(F, E)
$$

is an isomorphism for all covers $F \rightarrow R X$.

We defined $E$ as the full subcategory of $\left(X^{\mathrm{op}}, S\right)$ consisting of all the sheaves. Evidently every representable functor is a sheaf (in the canonical topology) so that $R$ induces a functor, also denoted by $R: X \rightarrow E$.

Suppose $X^{\prime} \rightarrow X$ is a regular epimorphism. We do not at this point assume that it is universally so, except for the supposition that the fibre square $X^{\prime} \times{ }_{X} X^{\prime}$ exists. Let $F$ be the image (pointwise) of $R X^{\prime} \rightarrow R X$. Then $R X^{\prime} \rightarrow$ $F$ is a regular epimorphism since in the functor category every epimorphism is. This means that

$$
R X^{\prime} \times_{F} R X^{\prime} \rightarrow R X^{\prime} \rightarrow F
$$

is a coequalizer. But $F \rightarrow R X$ a monomorphism implies that

so that

$$
R X^{\prime} \times_{F} R X^{\prime}=R X^{\prime} \times_{R X} R X^{\prime} \cong R\left(X^{\prime} \times_{X} X^{\prime}\right),
$$

$$
R\left(X^{\prime} \times_{X} X^{\prime}\right) \rightrightarrows R X^{\prime} \rightarrow F
$$

is a coequalizer. Then for all functors $F^{\prime}$,

$$
\left(F, F^{\prime}\right) \rightarrow\left(R X^{\prime}, F^{\prime}\right) \rightrightarrows\left(R\left(X^{\prime} \times_{X} X^{\prime}\right), F^{\prime}\right)
$$

is an isomorphism. In particular that is true for all $F^{\prime}=R Y$. On the other hand, since $R$ is full and faithful and $X^{\prime} \times{ }_{X} X^{\prime} \rightrightarrows X^{\prime} \rightarrow X$ is a coequalizer, we see that

$$
(R X, R Y) \rightarrow\left(R X^{\prime}, R Y\right) \rightrightarrows\left(R\left(X^{\prime} \times_{X} X^{\prime}\right), R Y\right)
$$

is an equalizer for all $Y \in X$. By the uniqueness of equalizers we see that $(R X, R Y) \cong(F, R Y)$ by an isomorphism such that

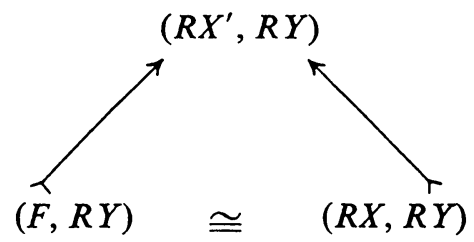


commute, which is only possible if it is induced by the natural map

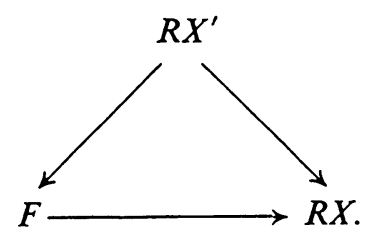

If $X^{\prime} \rightarrow X$ is a universal regular epimorphism, this analysis will remain the same under the pullback of any map $X_{1} \rightarrow X$ and we see that $F \mapsto R X$ is a cover. In that case, we have that

$$
(R X, E) \rightarrow\left(R X^{\prime}, E\right) \rightrightarrows\left(R X^{\prime} \times_{R X} R X^{\prime}, E\right)
$$

is an equalizer for every $E \in E$, which means that $R X^{\prime} \rightarrow R X=R\left(X^{\prime} \rightarrow X\right)$ is a regular epimorphism in $E$. (In any topos, every epimorphism is universally regular.) On the other hand, if $f$ is not a universal regular epimorphism but its pullback with every map exists, then we could easily see that $R f$ is not an epimorphism. The upshot of this is

Proposition 1. The canonical embedding $R: X \rightarrow E$ preserves universal regular epimorphisms; if $\boldsymbol{X}$ has pullbacks it also reflects them.

For general $\boldsymbol{X}$, it might happen that $R f$ is an epimorphism when $f$, for lack of enough pullbacks, is not a universal regular epimorphism. For example $f$ might be a split epimorphism-a property preserved by every functor. Accordingly, we would like to propose a new

Definition 1. A map $f$ is called a universal regular epimorphism if $R f$ is an epimorphism, where $R: X \rightarrow E$ is the Yoneda embedding of $X$ into the category of sheaves for the canonical topology on $X$.

Definition 2. A functor is called exact if it preserves all finite limits and universal regular epimorphisms. It is called reflexively exact if, in addition, it reflects finite limits and universal regular epimorphisms.

\section{The embedding.}

THEOREM A. Let $\boldsymbol{X}$ be a small category. Then there is a small category $C$ and a full reflexively exact embedding $U: X \rightarrow(C, S)$.

THEOREM B. Let $X$ be a small category. Then there is a (discrete) set $I$ and a functor $U: X \rightarrow S^{I}$ which is faithful and reflexively exact.

THEOREM C. Let $\boldsymbol{X}$ be a small category. Then there is a family $\left\{U_{i}\right\}$, $i \in I$ of exact set-valued functors on $\boldsymbol{X}$ which collectively are faithful and. reflect finite limits and universal regular epimorphisms.

Proof of Theorem A. The embedding $R: X \rightarrow E$ is full and exact. 
Since it is full, it clearly reflects finite limits and it reflects universal regular epimorphisms in accordance with Definition 1. According to (III, 1.2) of [Barr], $E$ has a full exact embedding into a (set-valued) functor category. Such an embedding evidently reflects finite limits and regular epimorphisms. But in a topos-including in a functor category-the classes of epimorphisms, regular epimorphisms and universal regular epimorphisms coincide. Hence it reflects exactness.

It is, of course, trivial that Theorem $A \Rightarrow$ Theorem $B \Rightarrow$ Theorem $C$.

3. An application. We illustrate the kind of diagram chasing argument possible with the following, which is a theorem of [Grothendieck] when the standard definition of universal regular epimorphism is used. It is also proved in [Barr] (III, 6.10) when the category is regular.

Proposition 2. Consider the diagram

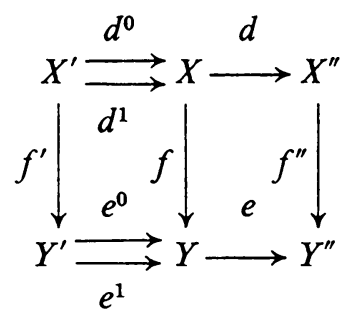

Suppose that

(i) $d$ is a universal regular epimorphism;

(ii) $d^{0}, d^{1}$ are the kernel pair of $d$;

(iii) $e^{0}, e^{1}$ are the kernel pair of $e$;

(iv) $f \cdot d^{0}=e^{0} \cdot f^{\prime} ; f \cdot d^{1}=e^{1} \cdot f^{\prime} ; f^{\prime \prime} d=e f$;

(v) the square

is a pullback.

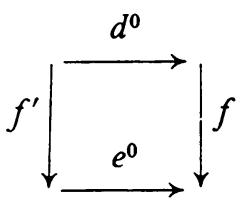

Then the square

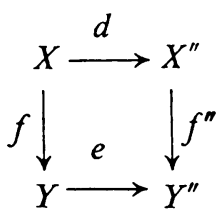

is a pullback.

Proof. Apply Theorem C and prove it for sets. 


\section{REFERENCES}

M. Barr, "Exact categories," in Exact categories and categories of sheaves, Lecture Notes in Math., no. 236, Springer-Verlag, Berlin and New York, 1971.

A. Grothendieck, Techniques de construction en géométrie analytique, Séminaire Henri Cartan, 1960/61, fasc. 1, Exposé 11, Ecole Normale Supérieure, Secrétariat mathématique, Paris, 1962. MR 26 \#3562.

M. Demazure, Topologies et faisceaux, Séminaire de géométrie algébrique 1963/64, fasc. 1, Exposé 4, Inst. Hautes Êtudes Sci., Paris, 1963/64. MR 34 \#7517.

Department of Mathematics, McGill University, Montreal, Quebec, Canada 